This item was submitted to Loughborough's Institutional Repository (https://dspace.lboro.ac.uk/) by the author and is made available under the following Creative Commons Licence conditions.

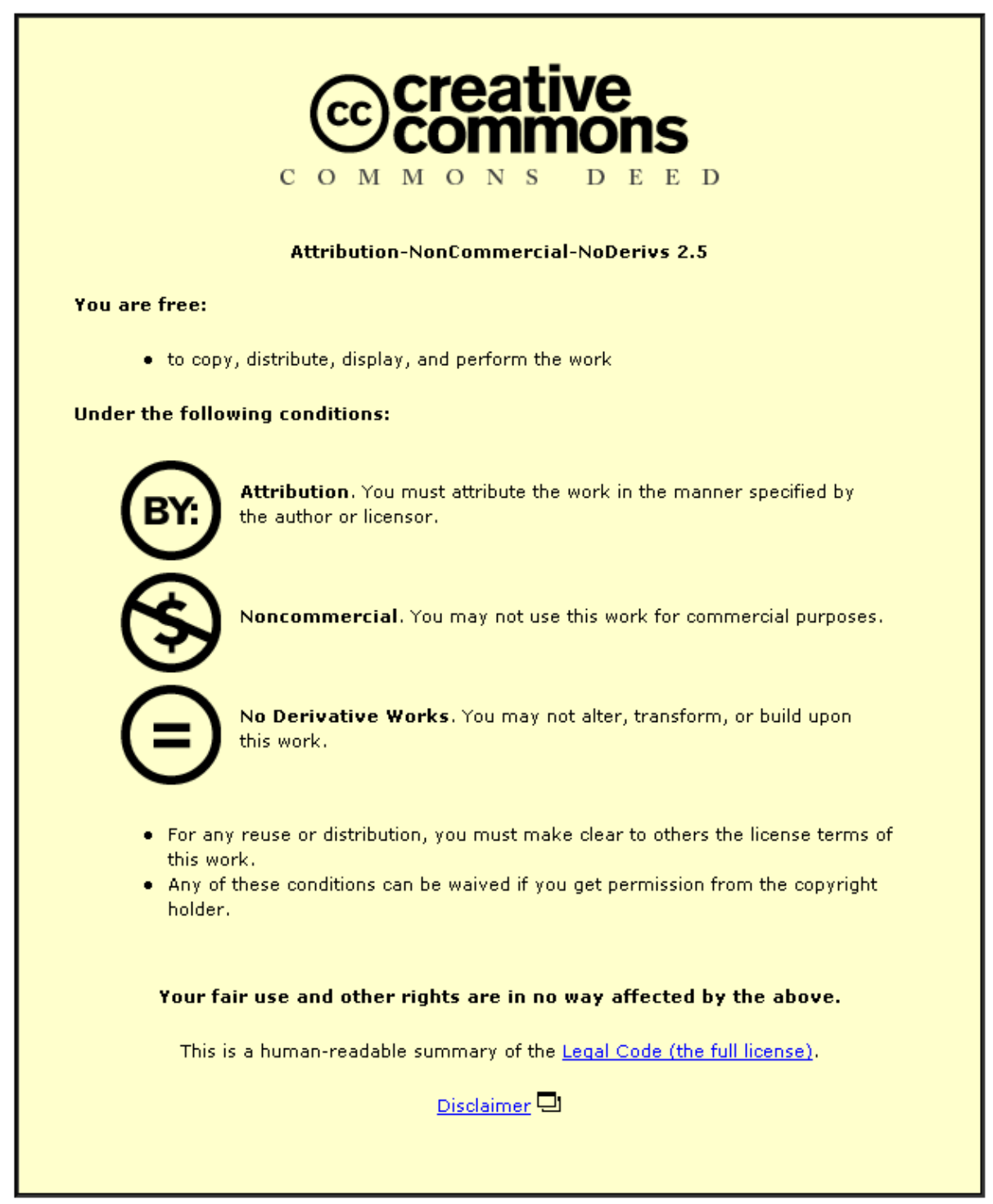

For the full text of this licence, please go to: http://creativecommons.org/licenses/by-nc-nd/2.5/ 
Ms. No. [Z485]

Angewandte Chemie International Edition, $\underline{49}$, 3006-3009 (2010)

Submitted 27 Jan 2010 Accepted 19 Feb 2010 Published Online 15 Mar 2010

The institutional repository is http://hdl.handle.net/2134/6067

The final publication is at http://www3.interscience.wiley.com/

The final publication DOI is DOI: 10.1002/anie.201000485

Copyright 2010 Wiley-VCH Verlag GmbH \& Co. KGaA, Weinheim.

This final manuscript version published by kind permission of Wiley-VCH Verlag GmbH \& Co. KGaA

\section{Selective Knockout of Gold Active Sites}

\section{Anna Maria Nowicka ${ }^{2}$, Ulrich Hasse ${ }^{1}$, Gustav Sievers ${ }^{1}$, Mikolaj Donten $^{2}$, Zbigniew Stojek ${ }^{2}$, Stephen Fletcher ${ }^{3}$, and Fritz Scholz ${ }^{1}$.}

${ }^{1}$ Institut für Biochemie, Universität Greifswald, Felix-Hausdorff-Str. 4, 17487

Greifswald (Germany)

${ }^{2}$ Dept. of Chemistry, Warsaw University u. Pasteura 1, 02-093 Warsaw (Poland)

${ }^{3}$ Department of Chemistry, University of Loughborough, Loughborough, LE11 3TU (UK)

\section{KEYWORDS}

Electrochemistry $\bullet$ gold $\bullet$ kinetics $\bullet$ radicals $\bullet$ voltammetry 


\section{ABSTRACT}

It has long been known that defects on a gold surface play an important role in electrocatalysis, but the precise mechanism has always been unclear. This work indicates that the defect sites provide partially filled $d$-orbitals that stabilize freeradical intermediates. Strong evidence for this hypothesis is that the sites can be selectively knocked out by treatment with $\mathrm{OH} \bullet$ radicals generated by Fenton's reagent. The knockout effect is demonstrated using oxygen reduction, hydrogen reduction, and the redox electrochemistry of hydroquinone.

\section{INTRODUCTION AND RESULTS}

Electrochemists commonly seek two diametrically opposed goals: (i) the activation of electrode surfaces, in order to overcome kinetic barriers and accelerate reactions, and (ii) the deactivation of electrode surfaces, in order to prevent unwanted reactions such as corrosion. It has long been known that the activity of electrodes (and of heterogeneous catalysts) is associated with surface defects [1-9]; platinum black is a familiar example [10]. In contrast, deactivation is typically achieved by "brute-force" methods such as total passivation $[11,12]$.

Recently, we demonstrated that the hydroxyl radicals $(\mathrm{OH} \bullet)$ of Fenton's reagent could remove self-assembled monolayers (SAMs) from gold electrodes [13], and surprisingly — could also dissolve asperities, thus leading to very smooth surfaces [14]. Herein we report that gold electrodes prepared by treatment with Fenton's reagent are also catalytically deactivated, almost certainly because the active sites on gold are selectively knocked out by the attack of the hydroxyl radicals. Unlike crystallo-graphically perfect gold surfaces, which have fully filled $d$ orbitals, the active sites on gold are thought to have partially filled $d$ orbitals. These orbitals resemble platinum surfaces, which normally have half-filled $d$ orbitals, and therefore they are similarly capable of catalysing reactions that involve free-radical intermediates.

Figure 1 shows current-potential curves of a gold electrode before and after successive treatments with Fenton's reagent. The solution was saturated with oxygen and the reduction peak of dissolved oxygen is clearly seen. Figure 2 shows the dependences of the peak potential and the peak current as a function of the duration of hydroxyl radical attack. The $\mathrm{O}_{2}$ reduction potentials shift negatively, while the peak currents remain almost constant. The data are consistent with the selective disappearance of the reactive $\mathrm{Au}$ atoms with partially filled $d$ orbitals, leaving behind only the un-reactive Au atoms with fully filled $d$ orbitals. Oxygen reduction requires the stabilization of a superoxide-free radical intermediate, which, as dictated by the Pauli exclusion principle, can not be achieved with fully filled $d$ orbitals.

It is interesting to compare the effects of polishing with Fenton's reagent (i.e., hydroxyl radical attack) with those of electroplating. When a fresh gold layer is 
electroplated on a mechanically polished gold surface, a partial inhibition of oxygen reduction is observed, which is consistent with the "burying" of some active sites, but not full inhibition. Thus, when the mechanically polished Au electrode was gold plated in a solution containing $0.1 \mathrm{M} \mathrm{NaClO}_{4}$ and $1 \mathrm{~mm} \mathrm{KAu}(\mathrm{CN})_{2}$, the oxygen reduction peak potentials were scattered between -0.19 and $-0.22 \mathrm{~V}$ ( $\mathrm{vs}$. $\mathrm{Ag} / \mathrm{AgCl}$ ), that is, these peak potentials were more negative than in the case of a mechanically polished surface and less negative than for a surface polished with Fenton's reagent.

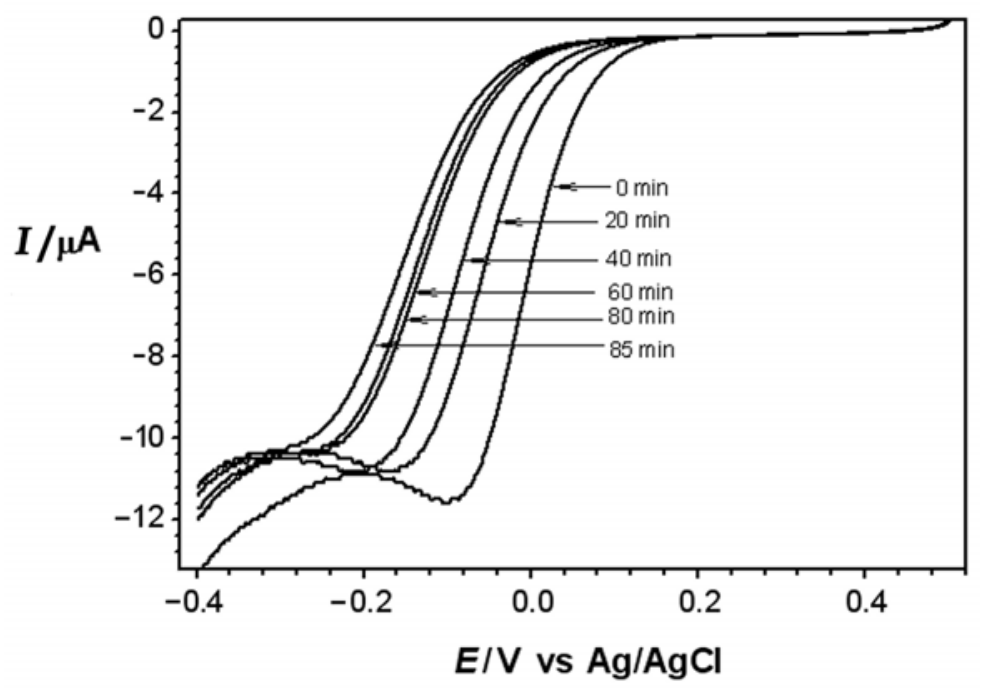

FIGURE 1. Voltammograms of reduction of $\mathrm{O}_{2}$ obtained in $0.1 \mathrm{M} \mathrm{H} 2 \mathrm{SO} 4$ after exposure of $\mathrm{Au}$ electrode to $\mathrm{OH} \bullet$ solution. Before experiments solution was saturated with oxygen. Conditions: scan rate $50 \mathrm{mV} / \mathrm{s}$; gold electrode diameter $(d=1.6 \mathrm{~mm})$; concentrations of $\mathrm{Fe}^{2+}$, EDTA and $\mathrm{H}_{2} \mathrm{O}_{2}: 1,1$ and $10 \mathrm{mM}$.

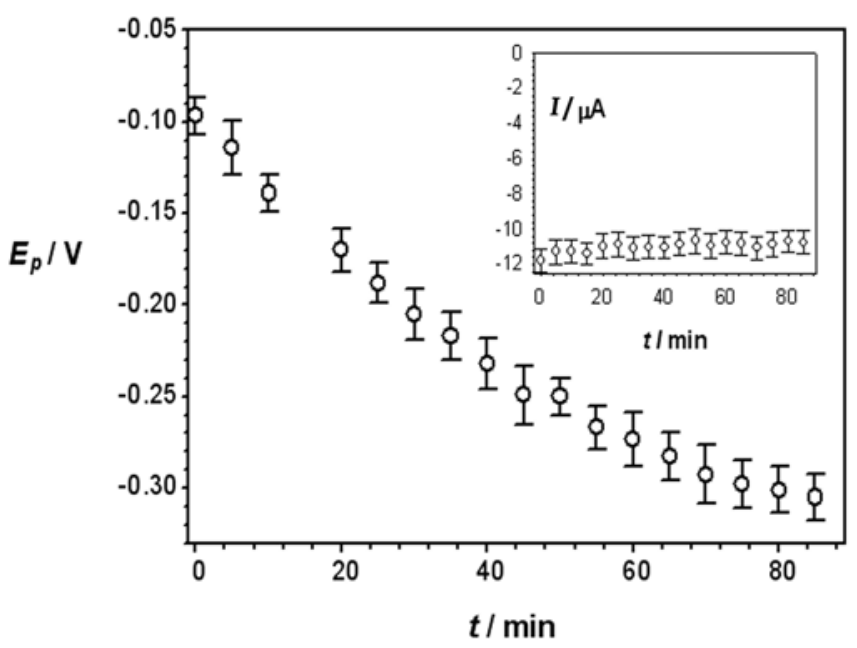

FIGURE 2. Peak potential of $\mathrm{O}_{2}$ reduction from saturated with oxygen $0.1 \mathrm{M} \mathrm{H}_{2} \mathrm{SO}_{4}$ versus time of reaction with $\mathrm{OH} \bullet$. Inset: Current reduction peak versus time of reaction with $\mathrm{OH} \bullet$. For other conditions see Figure 1. 
The hydrogen evolution reaction also proceeds via a radical intermediate, and, as expected, this reaction was also inhibited on the surface of gold electrodes treated with Fenton's reagent. In this case, the H+ reduction potential was shifted to negative potentials by about $45 \mathrm{mV}$ (results not shown).

The effect of hydroxyl radical $(\mathrm{OH} \bullet)$ attack on gold was probed using two different redox systems with different underlying kinetics, in order to provide critical proof for the catalytic deactivation. The first redox system, quinone-hydroquinone $(\mathrm{Q} / \mathrm{HQ})$, proceeds via a free radical intermediate [15], but the second redox system, $\mathrm{Ru}^{\mathrm{III}} / \mathrm{Ru}^{\mathrm{II}}$, does not. Figure 3 shows the cyclic voltammograms of Q/HQ obtained with a mechanically polished Au electrode before and after a series of treatments with Fenton's reagent. The plot of the logarithm of the corresponding normalized heterogeneous electron transfer rate constant versus time of interaction with the hydroxyl radicals is shown in the inset of Figure 3.

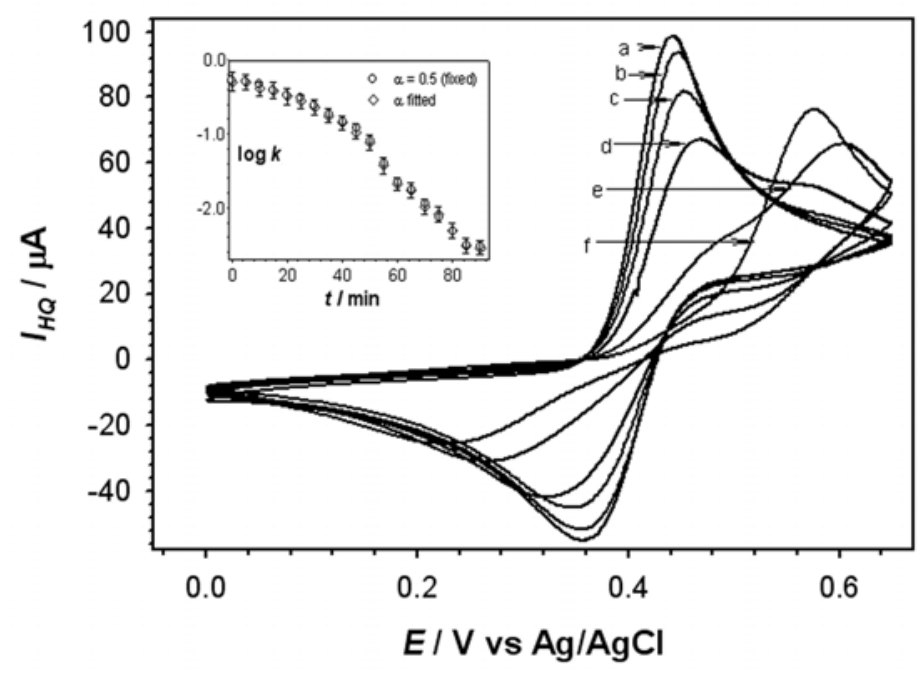

FIGURE 3. Cyclic voltammograms of $\mathrm{HQ}$ recorded in $0.1 \mathrm{M} \mathrm{HClO}_{4}$ containing 100 $\mathrm{mM} \mathrm{NaClO}_{4}$ after exposure of Au electrode to $\mathrm{OH} \bullet$ for (a) 0, (b) 10, (c) 20, (d) 40, (e) 60 and (f) 90 min. Inset: Mean electron transfer rate constant of HQ versus time of reaction with $\mathrm{OH} \cdot$. Conditions: scan rate $10 \mathrm{mV} / \mathrm{s}$; gold electrode diameter $(d=1.6$ $\mathrm{mm}) ; \mathrm{cHQ}=5 \mathrm{mM}$; concentrations of $\mathrm{Fe}^{2+}$, EDTA and $\mathrm{H}_{2} \mathrm{O}_{2}$ are 1,1 and $10 \mathrm{mM}$.

As expected, the $\mathrm{Q} / \mathrm{HQ}$ process became slower and slower as the electrode was exposed to hydroxyl radicals and the active sites were selectively knocked out. Chronocoulometry was used to exclude the involvement of any adsorption phenomena. In contrast, the $\mathrm{Ru}{ }^{\mathrm{III}} / \mathrm{Ru}^{\mathrm{II}}$ system did not show any changes in peak potential or peak current during attack by hydroxyl radicals; that is, the rate of electron transfer was unaffected within experimental error. Evidently, the active sites that are removed by polishing with Fenton's reagent are precisely those that stabilize free-radical intermediates. Upon attack by hydroxyl radicals, the open-circuit potentials (OCPs) of the gold electrodes were systematically shifted to more positive potentials: When the electrodes were reduced after each radical attack at $0.5 \mathrm{~V}$ versus 
$\mathrm{Ag} / \mathrm{AgCl}$ for $30 \mathrm{~s}$ (to obtain an oxide-free Au surface), the OCPs shifted from +0.232 $\mathrm{V}$ before treatment to $+0.247 \mathrm{~V}$ after 50 min of treatment with hydroxyl radicals. The effect is small but reproducible, however the complex interplay between oxygen or proton reduction and gold oxidation makes precise interpretation problematic.

The decrease of the rates of catalysed reactions on gold (and possibly other metals) by selective knockout of active sites is a new concept that may have considerable implications for corrosion protection. The contact of Au with less noble metals is responsible for much unwanted corrosion behaviour, in particular in the field of electronics, because $\mathrm{Au}$ acts as a galvanic half-cell where $\mathrm{O}_{2}$ may be reduced (or $\mathrm{H}+$ in some cases). An effective means of inhibiting $\mathrm{O}_{2}$ reduction and $\mathrm{H}+$ reduction in these circumstances without blocking the surface of the gold is clearly desirable. It has long been known that surface defects (i.e., any deviations from the structure of low-index planes) play an important role in electrocatalysis $[16,17]$, but the precise mechanism has always been unclear. The present work provides very strong evidence that surface defects are the loci of partially filled $d$ orbitals that can stabilize freeradical intermediates.

A simple proof is that these sites can be selectively knocked out by treatment with hydroxyl radicals $(\mathrm{OH} \bullet)$ generated by Fenton's reagent. Furthermore, since partially filled $d$ orbitals are responsible for electrocatalysis in the case of reactions that occur via radical intermediates, their removal is predicted to slow down the rates of $\mathrm{O}_{2}, \mathrm{H}+$, and $\mathrm{Q}$ reduction reactions, but not the $\mathrm{Ru}^{\mathrm{III}}$ reduction reaction; this behaviour is precisely what is observed. The need for orbital matching prior to electron transfer is emerging as a major theme of electrochemistry, and the theory is discussed in a related paper [18].

\section{EXPERIMENTAL}

Cyclic voltammetry (CV) was performed with an Autolab, model 12 potentiostat (Eco-Chemie, Utrecht) using a three-electrode system. A gold disc electrode (1.6 mm radius; BAS, Kenilworth) was used as the working electrode, a KCl-saturated $\mathrm{Ag} / \mathrm{AgCl}$ electrode served as the reference electrode, and a platinum wire was used as the auxiliary electrode. The solutions of Fenton's reagent: $\left(\mathrm{NH}_{4}\right)_{2} \mathrm{Fe}\left(\mathrm{SO}_{4}\right)_{2} \bullet 6\left(\mathrm{H}_{2} \mathrm{O}\right)(1$ $\mathrm{mm}$; Merck), ethylenediaminetetraacetate (EDTA; $1 \mathrm{~mm}$, Merck), acetate buffer $(0.01$ $\mathrm{M}, \mathrm{pH}$ 4.7), and $\mathrm{H}_{2} \mathrm{O}_{2}(10 \mathrm{~mm})$ were always freshly prepared. $\mathrm{H}_{2} \mathrm{O}_{2}$ was added immediately before starting the experiments. The surface of the working electrode was polished with 1.0 and $0.3 \mathrm{~mm} \mathrm{Al}_{2} \mathrm{O}_{3}$ on a wet pad before the measurements. After each polishing, the electrode was rinsed with a direct stream of water (Milli-Q, Millipore, conductivity of ca. $0.056 \mu \mathrm{S} / \mathrm{cm}$ ), and the electrode was dried. Then the electrode was cycled between $-0.3 \mathrm{~V}$ and $+1.5 \mathrm{~V}$ (vs. $\mathrm{Ag} / \mathrm{AgCl}$ ) in $\mathrm{H}_{2} \mathrm{SO}_{4}(0.1 \mathrm{M})$ until a stable voltammogram typical of a clean gold electrode was obtained [19]. Then the electrode was washed with water and exposed to freshly prepared solutions of Fenton's reagent for 5 minute intervals. The exposure was repeated so that the overall exposure time ranged from 5 to $90 \mathrm{~min}$. The reaction of Fenton's reagent with the gold electrode was terminated by removing the electrode from the solution of 
Fenton's reagent and washing it with water. The electrocatalytic activity of gold electrodes was probed with $\mathrm{O}_{2}$ in acidic solution; hydroquinone $(5 \mathrm{~mm}$, Fluka, purity $99 \%$ ), and ruthenium(III) hexamine (5 mm solution in $0.1 \mathrm{M} \mathrm{NaClO}_{4}$; Aldrich, purity $98 \%$ ). The reported electron transfer rate constant was normalized according to the following formula:

$$
k_{\mathrm{s}, \text { norm }}=k_{\mathrm{s}} \sqrt{\frac{\tau}{D_{\mathrm{r}}}}
$$

where $k_{\mathrm{s}}$ is the electron transfer rate constant, $k_{\mathrm{s}, \text { norm }}$ is the normalized electron transfer rate constant, $D_{\mathrm{r}}$ is the diffusion coefficient of the reactant, and $\tau$ is the characteristic time parameter of the technique.

The time parameter for voltammetry takes the form

$$
\tau=\frac{R T}{n F v}
$$

where $v$ is the scan rate. The parameter $k_{\mathrm{s}, \text { norm }}$ was determined by fitting simulated voltammograms to the experimental voltammograms using GEPES software.

\section{ACKNOWLEDGEMENTS}

A.M.N. acknowledges kind support by DAAD, Bonn. G.S. acknowledges support by the Alfried Krupp Wissenschaftskolleg Greifswald. Research Funded by: DAAD, Alfried Krupp Wissenschaftskolleg Greifswald.

\section{REFERENCES}

[1] G. Ertl, Angew. Chem. 2008, 120, 3578-3590; Angew. Chem. Int. Ed. 2008, 47, 3524-3535.

10.1002/ange.200800480; 10.1002/anie.200800480.

[2] H.-J. Freund, Angew. Chem. 1997, 109, 444-468; Angew. Chem. Int. Ed. 1997, $36,452-475$.

10.1002/ange.19971090504

[3] C. E. Banks, A. Crossley, C. Salter, S. J. Wilkins, R. G. Compton, Angew. Chem. 2006, 118, 2595-2599.

10.1002/ange.200600033.

[4] L. D. Burke, Gold Bull. 2004, 37, 125-135.

[5] D. L. Burke, L. M. Hurley, J. Solid State Electrochem. 2002, 6, 101-110.

$10.1007 / \mathrm{s} 100080100205$.

[6] L. D. Burke, J. M. Moran, P. F. Nugent, J. Solid State Electrochem. 2003, 7, 529538 .

10.1007/s10008-003-0359-y. 
[7] A. J. Ahern, L. C. Nagle, D. L. Burke, J. Solid State Electrochem. 2002, 6, 451462.

10.1007/s10008-001-0263-2.

[8] L. D. Burke, A. P. O Mullane, J. Solid State Electrochem. 2000, 4, 285-297.

[9] L. D. Burke, M. A. Murphy, J. Solid State Electrochem. 2001, 5, 43-49.

$10.1007 / \mathrm{s} 100089900095$.

[10] U. Schröder, J. Nießen, F. Scholz, Angew. Chem. 2003, 115, 2986-2989;

Angew. Chem. Int. Ed. 2003, 42, 2880-2883.

10.1002/ange.200350918; 10.1002/anie.200350918.

[11] W. A. Badawy, F. M. Al-Kharafi, Corros. Sci. 1997, 39, 681-700.

10.1016/S0010-938X(97)89336-5.

[12] L. Q. Zhu, F. Yang, Corros. Prot. 2006, 27, 503-507.

[13] F. Scholz, G. L pez de Lara Gonz lez, L. Machado de Carvalho, M. Hilgemann,

Kh. Z. Brainina, H. Kahlert, R. S. Jack, D. T. Minh, Angew. Chem. 2007, 119, 8225

8227; Angew. Chem. Int. Ed. 2007, 46, 8079-8081.

10.1002/ange. $200702690 ; 10.1002$ /anie. 200702690.

[14] A. M. Nowicka, U. Hasse, M. Hermes, F. Scholz, Angew. Chem. 2010, 122,

1079-1081; Angew. Chem. Int. Ed. 2010, 49, 1061-1063.

10.1002/anie.200906358.

[15] X. Huang, S. Wang, X. Shan, X. Chang, N. Tao, J. Electroanal. Chem., DOI: $10.1016 /$ j.jelechem.2009.12.027.

[16] J. O M. Bockris, A. K. N. Reddy, M. Gamboa-Aldeco, Modern Electrochemistry, Vol. 2A, 2nd ed., Kluwer, New York, 2000, p. 1275.

[17] M. T. M. Koper, Faraday Discuss. 2009, 140, 11-24.

10.1039/b812859f.

[18] S. Fletcher, J. Solid State Electrochem. 2010, 14, 705-739.

10.1007/s10008-009-0994-z.

[19] H. O. Finklea, S. Avery, M. Lynch, T. Furtsch, Langmuir 1987, 3, 409-413.

10.1021/la00075a024. 